\title{
Circadian rhythms of locomotor activity in Ansell's mole-rat: are mole-rat's clocks ticking?
}

\author{
J. L. de Vries ${ }^{1}$, M. K. Oosthuizen ${ }^{1}$, A. M. Sichilima ${ }^{1}$ \& N. C. Bennett ${ }^{1}$ \\ 1 Department of Zoology and Entomology, Mammal Research Institute, University \\ of Pretoria, Pretoria, South Africa
}

Correspondence

M. K. Oosthuizen, Department of Zoology and Entomology, Mammal Research Institute, University of Pretoria, Pretoria 0002, South Africa.

Email: moosthuizen@zoology.up.ac.za

\begin{abstract}
Circadian rhythms of locomotor activity have been investigated in several African mole-rat species. Even though mole-rats spend most of their lives in underground burrows devoid of light, studies have shown that they do possess circadian rhythms to some extent. We investigated the circadian rhythms of locomotor activity in 11 male Ansell's mole-rats Fukomys anselli from Zambia. In order to determine whether these animals can entrain to light and have endogenous rhythms, they were subjected to different light regimes: first, $12 \mathrm{~h}$ light/ $12 \mathrm{~h}$ dark, followed by constant darkness, then returned to $12 \mathrm{~h}$ light/ $12 \mathrm{~h}$ dark, which was later inversed to $12 \mathrm{~h}$ dark/12 h light. Only two individuals displayed arrhythmic activity patterns whereas the other nine $(81.8 \%)$ exhibited entrainment of their activity to the light regimes. Locomotory activity of Ansell's mole-rat was predominantly during the dark phase in all light regimes. During constant darkness (DD), only five individuals (45.5\%) displayed very weak circadian rhythms that free ran but became more indistinct towards the end of the cycle. Under the second LD light cycle, $90.1 \%$ of the animals were active during the night phase of the cycle and when placed under an inverse light cycle, seven individuals still displayed activity predominantly during the dark phase. In conclusion, these results suggest that Ansell's mole-rat does have a weak circadian clock and is able to perceive light and entrain to light cycles.
\end{abstract}

\section{Introduction}

Biological processes in all eukaryotes are regulated by endogenous rhythms that may vary with time from seconds to days (Reuss, 1996). By far the most common rhythmic events are circadian rhythms, which are expressed daily, and found in virtually all organisms examined to date (Goldman, 1999). Without external cues, the circadian rhythm free runs with a period $(\tau)$ close to the 24-h period of the day (Moore, 1983). In mammals, the circadian system is regulated by the suprachiasmatic 
nucleus (SCN) that is found in the anterior hypothalamus (De Vries \& De Vries, 1995; Reuss, 1996). Photic signals are received from the retina and subsequently relayed to the SCN, which, in turn, acts as a master clock and entrains to these external cues otherwise known as zeitgebers (Reuss, 1996; Van Esseveldt, Lehman \& Boer, 2000). Light is the most common cue that animals entrain to - this process is known as photoentrainment (for an overview, see Moore, 1983). It has been shown that social cues may also function as zeitgebers in Octodon degus, whereby animals synchronize their individual rhythms with the other animals in the colony (Goel \& Lee, 1995). Circadian rhythms function to synchronize the activity of the organism with both the outside environment and the internal biological events (Roenneberg $\&$ Foster, 1997). Synchronizing internal metabolic and physiological processes as well as behavioural pattern to environmental functions increases fitness (Sharma, 2003). Circadian rhythms provide the animal with the ability to anticipate changes in the environment.

African mole-rats from the family Bathyergidae are rodents endemic to sub-Saharan Africa. They live in burrow systems and rarely, if ever, emerge above the ground. African mole-rats show several adaptations to their underground lifestyle that include small eye size and a regressed visual system (Bennett \& Faulkes, 2000).

Lovegrove \& Papenfus (1995) and Oosthuizen, Cooper \& Bennett (2003) investigated circadian rhythmicity in the solitary Cape mole-rat Georychus capensis, a species commonly found above ground and possessing proportionally larger eyes than Fukomys anselli. The Cape mole-rat was found to have a well-defined endogenous rhythm of locomotory activity and a well-defined free-running period $(\tau)$ under constant conditions. The ability to entrain to a $12 \mathrm{~h} \mathrm{light} / 12 \mathrm{~h}$ dark cycle illustrated that the mole-rat could use light as a major entraining agent (Lovegrove \& Papenfus, 1995). In contrast, many of the social species are entirely subterranean and rarely, if ever, are found on the surface. As a consequence, it has been hypothesized that species of the social mole-rat are probably less sensitive to light entrainment.

Interestingly, to date, many of the African mole-rat species that occupy higher latitudes have been found to exhibit marked endogenous rhythms of locomotor activity that can be entrained by light. Hence, the common mole-rat Cryptomys hottentotus hottentotus, the Lesotho mole-rat Cryptomys hottentotus sp., the Mashona mole-rat Fukomys darlingi, Natal mole-rat Cryptomys natalensis and the Damaraland mole-rat Fukomys damarensis, all show endogenous rhythms of locomotor activity that can be entrained to light/dark cycles. All species studied to date have some individuals that allow their endogenous rhythm to free run under constant conditions (Oosthuizen et al., 2003; Hart et al., 2004; Vasicek et al., 2005; Schöttner et al., 2006).

The blind mole-rat Spalax ehrenbergi of the family Spalacidae has been used extensively as a model to investigate circadian rhythms in subterranean mammals (Haim et al., 1983; Vuillez et al., 1994; Ben-Shlomo, Ritte \& Nevo, 1995; Goldman et al., 1997; Tobler et al., 1998; Oster et al., 2002). These mole-rats possess small subcutaneous, atrophied eyes, but are still able to entrain to light cycles (Cooper, Herbin \& Nevo, 1993). This indicates that they have the ability to distinguish between light and dark, even though the region of the brain associated with image forming is extremely reduced (87-93\%) (Cooper et al., 1993). 
However, African mole-rats from the family Bathyergidae do not appear to be blind in the physiological sense, because they possess basic visual capabilities (Němec, Burda $\&$ Peichl, 2004). It has been suggested that Ansell's mole-rats have better visual capabilities than that of S. ehrenbergi (Cernuda-Cernuda et al., 2003; Němec et al., 2004). Because the basic visual pathways are intact in African mole-rats, one would also expect that the pathways involved in the circadian rhythmicity of the mole-rat would also be functional. On average, the eyes of Cryptomys species are $2.1 \mathrm{~mm}$ in diameter and set in the Harderian glands (Negroni, Bennett \& Cooper, 2003). The visual systems of Ansell's mole-rat, F. anselli, appear to have functional subsystems involved in photoperiod, form, brightness and movement analysis, while those involved in coordination of visuomotor reflexes are severely reduced (Němec et al., 2004). Hence, all evidence suggests that Ansell's mole-rat has the capability to distinguish between light and dark.

Experiments undertaken to investigate whether African mole-rats are nocturnal or diurnal have proven to be very inconclusive. In a study by Hart et al. (2004) on the Natal mole-rat (Cryptomys hottentotus natalensis), some of the animals were nocturnal, some diurnal and a few showed no apparent pattern. Georychus capensis exhibited predominantly nocturnal activity patterns when exposed to a $12 \mathrm{~h}$ light/12 h dark cycle (Lovegrove \& Papenfus, 1995; Oosthuizen et al., 2003). In contrast to G. capensis, F. damarensis showed a strong preference for diurnal activity (Lovegrove, Heldmaier \& Ruf, 1993).

Ansell's mole-rat F. anselli is a herbivorous African mole-rat that is endemic to the savannas of Zambia. The mole-rats occupy an extensive burrow system that is completely sealed from the surface. Ansell's mole-rats are social and colony sizes range from 2 to 25 animals, but averages around 12 animals. The subterranean lifestyle rarely provides opportunities for the mole-rats to receive exposure to external light cues. The colonies exhibit a marked reproductive skew in which a single female is responsible for procreation and litters are produced throughout the year (Burda, 1989).

Previous evidence suggests that social mole-rat species are able to perceive light (Oosthuizen et al., 2003; Hart et al., 2004; Vasicek et al., 2005; Schöttner et al., 2006); thus, we would predict that Ansell's mole-rats are able to entrain their locomotor activity to light cycles. Because these animals are found in an area where the environmental conditions are fairly constant (close to the equator), these animals were predicted to be unable to maintain a functional circadian clock and exhibit endogenous rhythmicity of locomotory activity.

\section{Materials and methods}

\section{Animals}

Experimental animals (Ansell's mole-rats F. anselli) were captured about $25 \mathrm{~km}$ west of Lusaka from February to April 2007. The trapping methods consisted of modified Hickman live traps (Hickman, 1979) placed at an opening to the burrow system, and trapping an animal in the burrow system by blocking the tunnel with a hoe. After 
capture, the animals were transported to the Republic of South Africa (Permit no.13/1/30/2/9/8-127), to the University of Pretoria (Permit no. CPB6 000036), where the experiments took place. The protocol was cleared by the Animal Use and Care Committee at the University of Pretoria (AUCC060719-020).

The adult male mole-rats were housed individually in plastic containers and provided with wood shavings and tissue paper as nesting material. They were fed ad libitum on chopped sweet potatoes and apples during the light phase of the cycle or with a red light when animals were on a DD cycle. Feeding was carried out at random times to ensure that the animals did not entrain to a specific feeding time. During the feeding times, the general health and condition of the animals was monitored. The cages were cleaned once each cycle was completed.

The experimental room was temperature controlled at $25 \pm 1^{\circ} \mathrm{C}$ and an extractor fan created a constant white noise to block out any other noises that might disturb the animals. Animal cages were spaced out under a fluorescent light source of c. 500 lx. The room was light controlled, which enabled us to create different light regimes for the experimental period.

\section{Experimental model}

We used 11 male mole-rats for the experiments, and they were kept in the same room and were thus exposed to the exact and same lighting regimes. Before commencing the experiments, animals were placed in constant darkness for 30 days to recreate their natural environment. After this initial 30-day period, they were placed under a $12 \mathrm{~h}$ light $/ 12 \mathrm{~h}$ dark cycle (8:00-20:00 h light) for 20 days to determine how well they entrain to light cycles. This was followed by a 20-day DD cycle in order to determine whether the mole-rats have endogenous free-running rhythms of locomotor activity. The animals were placed back onto a $12 \mathrm{~h}$ light/12 h dark cycle (8:00-20:00 h light) for a further 20 days to investigate how rapidly they could potentially re-entrain to the light cycle, before inversing the $12 \mathrm{~h}$ dark/12 $\mathrm{h}$ light cycle (8:00-20:00 h dark) for the final 20 days of the experiment to determine whether the activity shifts with the light cycle. Cages were cleaned when light cycles were changed, and animals were given the rest of the day to settle down from the disturbance.

\section{Activity measures}

An infrared captor [Quest PIR internal passive infrared detector; Elite Security Products (ESP), Electronic Lines, London, UK] was fitted above each of the 11 cages in which the animal was housed. These sensors were placed in the middle of each cage so as to detect any movement that the animal made in any area of the cage. A reading was taking once every minute. The activity measurements were captured by the program Vital View on a computer system (Vital View ${ }^{\mathrm{TM}}$; Minimitter Co. Inc., Sunriver, OR, USA; http://www.minimitter.com). 


\section{Data analyses}

Double-plotted actograms were created with Actiview Biological Rhythm Analyses 1.2 software (Minimitter Co., Inc.; http://www.minimitter.com). This gave a graphic representation of the activity patterns for each light cycle. Microsoft Excel was used to determine percentage activity either during the day or the night phase of the light regimes for each animal. From these percentages, a mean was worked out for each light regime and for all put together. This enabled us to determine the preferred period of activity for each animal.

\section{Results}

\section{General observations}

The mole-rats entrained to the light cycles and shifted their locomotory activity patterns during changes in the light cycle (Fig. 1). Two individuals (mole-rats 2 and 9) were arrhythmic and displayed no entrainment to any of the designated light cycles (Fig. 2). The rhythms displayed by the other nine individuals were not very robust and poorly defined in most of the mole-rats. Most of the locomotory activity occurred during the dark phase of the respective light regimes $(58.70 \pm 0.84 \%, \mathrm{n}=11)$. Activity was, however, not restricted only to the dark phase. Out of the 11 animals, only three had a higher percentage of activity during the light phase of the respective light cycles, while eight individuals had more than $50 \%$ of the activity confined to the dark phase. Variation in the time and amount of activity was observed between and within all the mole-rats. 


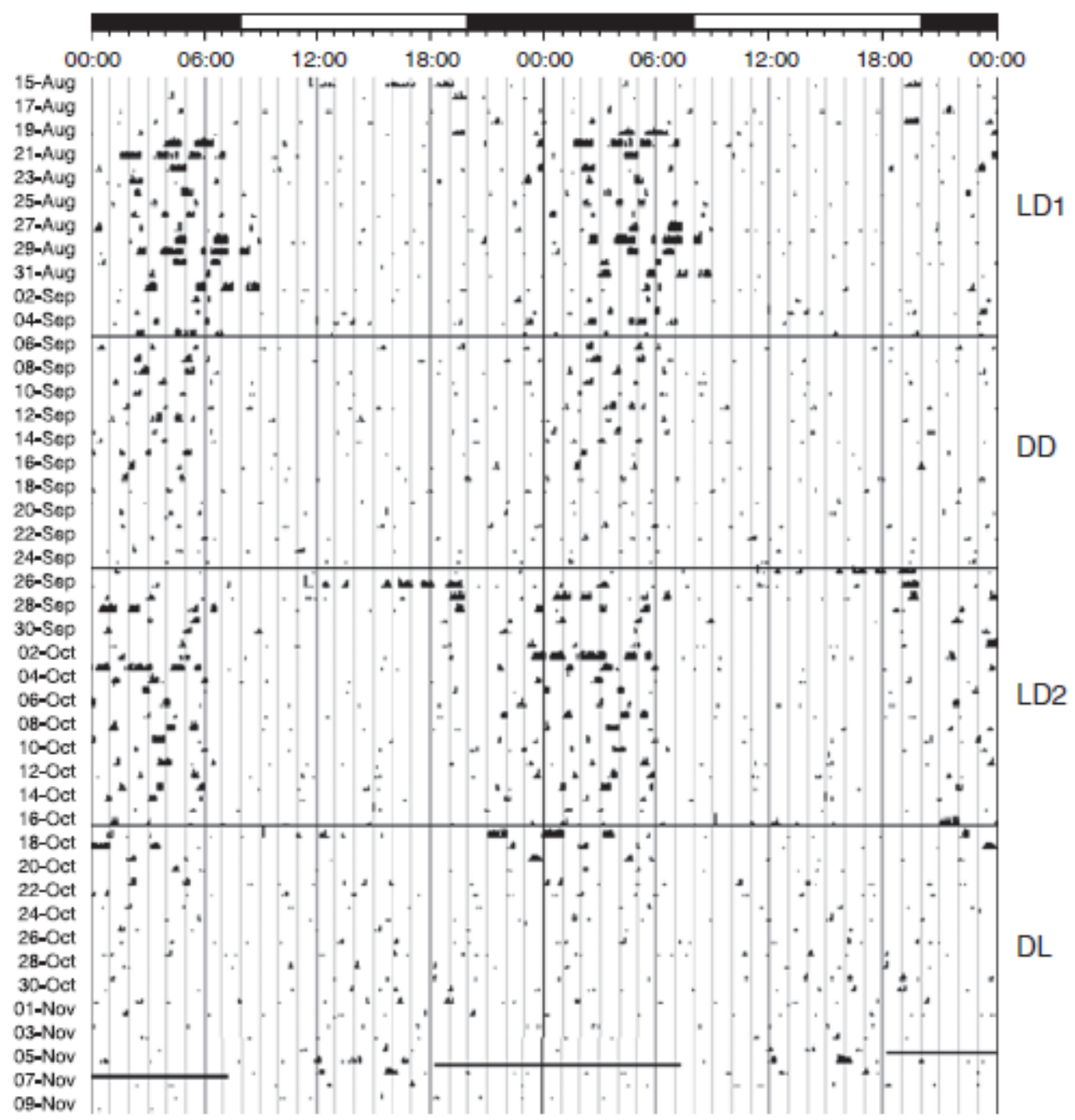

Figure 1 Actogram of the activity pattern of mole-rat number 8 . The individual shows entrainment to light during the two LD cycles, a poorly delineated endogenous rhythm during the DD cycle and a change in activity to the dark phase in the DL cycle. The cycles were in the order from top to bottom LD1, DD, LD2 and DL. The black and white bar at the top of the graph indicates the light and dark phase in the first two LD cycles, and the inverse last of the cycle. 


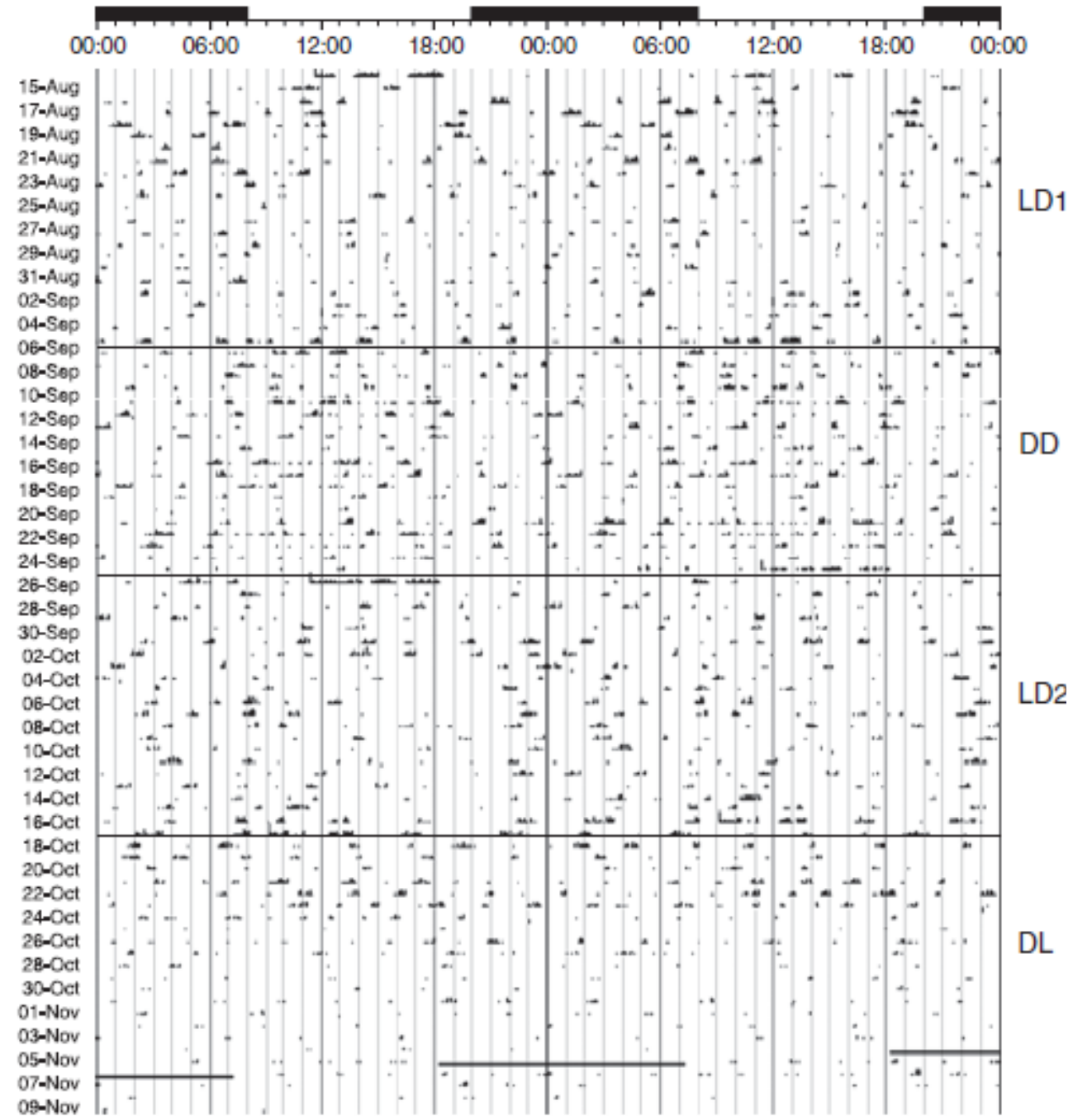

Figure 2 Actogram of activity patterns of mole-rat number 9. The individual shows an arrhythmic pattern of activity and no entrainment to any of the light regimes. The lines across the graph indicate the end of one cycle and the beginning of the next. The cycles were in the order from top to bottom LD1, DD, LD2 and DL. The black and white bar at the top of the graph indicates the light and dark phase in the first two LD cycles, and the inverse last of the cycle.

Two individuals (mole-rats 1 and 4) exhibited endogenous circadian rhythms during the DD regime of the experiment, which was the same as the period of activity during the dark phase of the first LD light regime. The period $(\tau)$ of this rhythm was close to $24 \mathrm{~h}$, but was not clearly defined or very robust.

\section{Photoperiod LD and constant dark DD light regimes}

Entrainment to the first LD cycle was observed in nine out of 11 mole-rats and animals showed a preference for the dark phase of the cycle $(60.15 \pm 1.32 \%, \mathrm{n}=11)$. Two individuals did not entrain and their locomotor activity patterns were arrhythmic (Fig. 2). Only three individuals (mole-rats 2, 4 and 7) displayed a preference for the light phase (Fig. 3). There was a large variation in the time of onset and offset of the 
locomotory activity rhythms both between individuals and within the same individual. This implies that the mole-rats do not entrain to the actual change of the light phase, but rather to the presence or absence of a light stimulus.

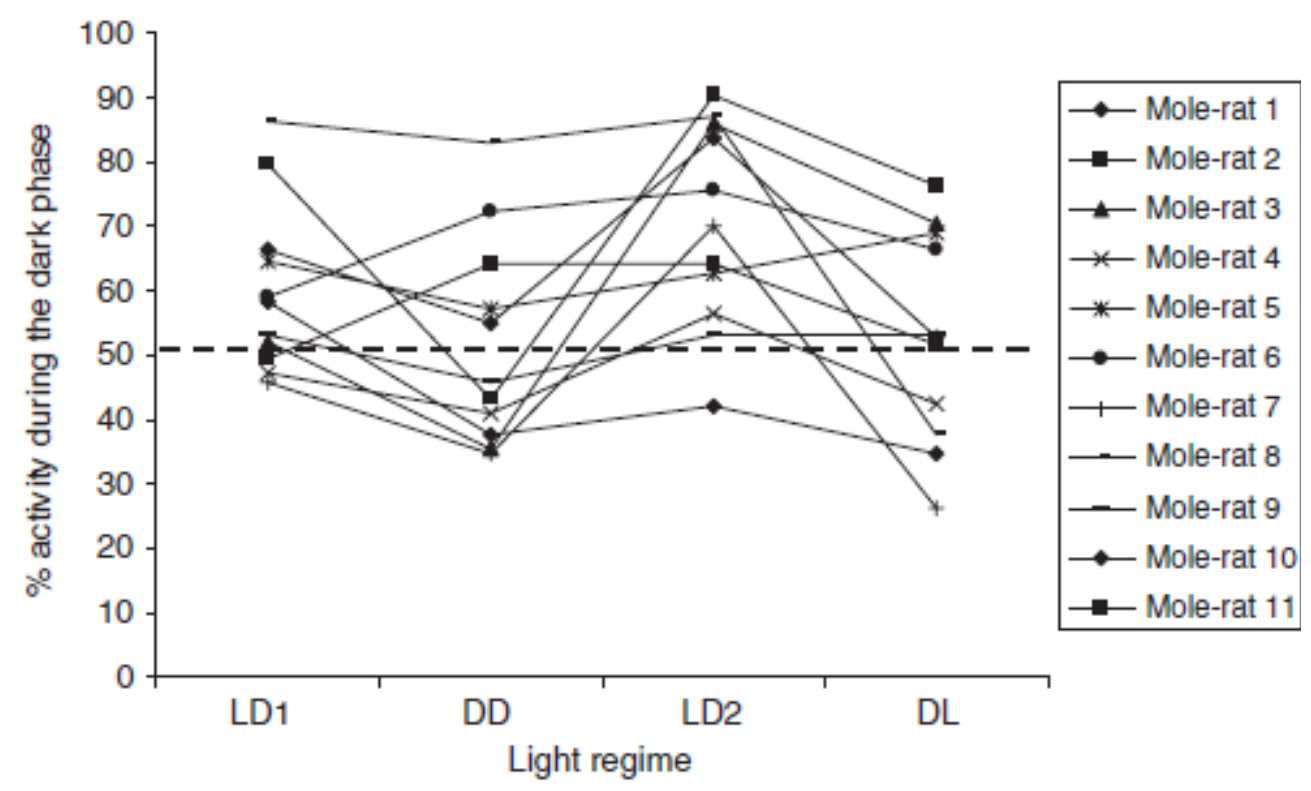

Figure 3 Mean percentage of activity for each mole-rat during the night phase of each light regime.

During the DD lighting regime, most individuals appeared to be arrhythmic. Five individuals followed the same activity pattern as observed in the previous cycle (Fig. 1), suggesting that they have endogenous rhythms of a length similar to those rhythms that are entrained. The rhythms degenerated a few days into the experiment. There was little to no drift in activity patterns of animals that exhibited a rhythm, implying that the endogenous circadian rhythm does indeed correspond with $\tau$ very close to $24 \mathrm{~h}$. Percentages of activity during the subjective day and night phases were almost equal (night $51.71 \pm 1.61 \%$ ). There were thus no preferences for any specific time of activity (Fig. 3). Only two animals had increased activity during this cycle (mole-rats 2 and 6). In four individuals, the activity pattern degenerated towards the end of the DD lighting regime, again indicating a weak coupling to the pacemaker (Fig. 1).

\section{Photoperiod LD and inverse photoperiod DL light regimes}

During the dark phase of the LD cycle following DD, all the individuals were more active than during the subjective night phase of the DD cycle $(70.13 \pm 1.59 \%)$, with most individuals (seven mole-rats) exhibiting an activity peak in the dark phase of this cycle that was higher than any other observed peaks in the study (Fig. 3). Locomotory activity increased in all but one individual (mole-rat 2), and in this individual activity declined by only $0.1 \%$. Only mole rat 1 displayed a preference for diurnal activity (58\% day active). 
Activity decreased during the night phase of the DL cycle $(52.8 \pm 1.64 \%)$. Of the 11 mole-rats, seven were still more active during the dark phase of the cycle, even though all of them had reduced activity (Fig. 3). Only two individuals (mole-rats 5 and 9) showed increased activity during the night phase of the DL cycle (Fig. 3).

\section{Discussion}

\section{Entrainment of activity}

Locomotor activity rhythms in F. anselli are not very well defined. Large variations in activity patterns occur both within and among individuals. Two individuals were arrhythmic, and did not entrain to any of the lighting regimes throughout the entire experiment (Fig. 2). Variabilities in activity patterns have been found in the Natal mole-rat, highveld mole-rat and the Lesotho mole-rat, which are all subspecies of C. hottentotus (Oosthuizen et al., 2003; Hart et al., 2004; Schöttner et al., 2006). In species more closely related to F. anselli, similar results have been found. The Damaraland mole-rat F. damarensis and Mashona mole-rat F. darlingi, both belonging to the genus Fukomys, have also been found not to exhibit strong, robust rhythms (Oosthuizen et al., 2003; Vasicek et al., 2005). This indicates that light may not function as the only or the most prominent zeitgeber in these subterranean species, but that other factors such as temperature and social cues may play a role. Lovegrove et al. (1993) found that when F. damarensis is placed under various lighting regimes, as a colony, it entrains its activity patterns to these light regimes. Bovet \& Oertli (1974) suggested that beavers Castor canadensis use social cues to synchronize their own activity patterns to that of the colony. In O. degus, it has been found that cues from conspecifics can influence the circadian rhythm when placed under an LD cycle (Goel $\&$ Lee, 1995). This suggests that further investigations should be conducted to determine the role that social cues play in entrainment, especially in subterranean species. Because mole-rats are rarely, if ever, exposed to light cues, it is possible that other cues such as social cues could play a more important role in the entrainment of activity patterns. The variation in activity may also be caused by a weak coupling between the pacemaker and the expressed rhythms, and because light cues do not reach the SCN from the retina (Oosthuizen et al., 2003; Vasicek et al., 2005).

\section{Endogenous circadian rhythms}

Five of the individuals that entrained to the first light cycle showed signs of circadian rhythms in the DD cycle (Fig. 1). There was no drift in locomotor activity, indicating that they have an endogenous rhythm with a period close to $24 \mathrm{~h}$, which is the same as that found in the Lesotho mole-rat (Schöttner et al., 2006). However, the rhythm was very weak, and this might be because of weak entrainment or a weak circadian rhythm. Oosthuizen et al. (2003) found endogenous rhythms in two social species of mole-rats, but they found that the same percentage of animals that entrained to the first light cycle displayed rhythms in the DD cycle as well. In F. anselli, only 55\% of the individuals that entrained to the LD cycle showed signs of circadian rhythms, and in these individuals the rhythms tended to fade away during the last part of the DD cycle. The remainder of the animals displayed arrhythmic activity patterns that further support a very weak rhythm. 
The subterranean environment that mole-rats occupy provides them with a constantly dark environment. In a constant environment, there is very little adaptive advantage of maintaining a circadian clock locomotor activity (Sharma, 2003). Because Ansell's mole-rat is not a seasonal breeder (Bennett \& Faulkes, 2000), it does not need to rely on external cues to indicate when the breeding season starts. Fukomys anselli is found close to the equator where temperature change is muted and small fluctuations in daylight hours occur across seasons. This again suggests that light cues may not play a crucial role in the entrainment of circadian rhythms because they no longer rely on changes from the above-ground environment.

\section{Temporal distribution of activity}

The majority of the mole-rats displayed activity mainly during the dark phase of all the light regimes; a few animals displayed diurnal activity. Some animals displayed nocturnal activity patterns in one cycle and then diurnal in another, while others had activity patterns that were fairly spread out across both day and night phases. These findings are similar to what Oosthuizen et al. (2003) have found in the two social mole-rat species, F. damarensis and Cryptomys hottentotus pretoriae. These species have been found to be diurnal and nocturnal, respectively. This suggests that social cues could play an important role in the entrainment of rhythms in social species. Because mole-rats are very rarely exposed to light cues due to their subterranean lifestyle, they should benefit more from entraining to social cues from the colony or temperature cues from the muted changes that take place in the burrow daily. It may be more beneficial for a colony of mole-rats to stagger their activity at different times of the day, such that they can continuously patrol the burrow system, close open entrances and forage.

\section{Conclusion}

The results suggest that the African mole-rat F. anselli has the ability to distinguish between light and dark and is capable of entraining to light cycles, albeit weakly. This is indicated by the fact that the mole-rats were predominantly active during the dark phase of the LD cycles, and further emphasized by the shift in activity the mole-rats exhibited when the LD cycle was inverted (Fig. 1). Activity was mainly during the dark phase of all the cycles (Fig. 3), even though a few individuals exhibited diurnal activity under some of the light regimes (Fig. 3). There was a very weak indication of endogenous circadian rhythmicity because a few individuals continued to express an activity pattern that free ran when placed under constant dark conditions. No phase shift in activity was exhibited during the DD phase, and the rhythms disintegrated after a few days in DD.

Therefore, we can conclude that Ansell's mole-rat is able to detect light and entrain to it; they do exhibit circadian rhythms but the rhythmicity is not very robust. 


\section{References}

- Bennett, N.C. \& Faulkes, C.G. (2000). African mole-rats ecology and eusociality. Cambridge, UK: Cambridge University Press.

- Ben-Shlomo, R., Ritte, U. \& Nevo, E. (1995). Activity pattern and rhythm in the subterranean mole rat superspecies Spalax ehrenbergi. Behav. Genet. 25, 239-245.

- Bovet, J. \& Oertli, E.F. (1974). Free-running circadian activity rhythms in free-living beaver (Castor canadensis). J. Comp. Physiol. 92, 1-10.

- Burda, H. (1989). Reproductive biology (behaviour, breeding and postnatal development) in subterranean mole-rats, Cryptomys hottentotus (Bathyergidae). Z. Saeugetierkd. 54, 360-376.

- Cernuda-Cernuda, R., García-Fernández, J.M., Gordijn, M.C.M., BoveeGeurts, P.H.M. \& DeGrip, W.J. (2003). The eye of the African mole-rat Cryptomys anselli: to see or not to see? Eur. J. Neurosci. 17, 709-720.

- Cooper, H.M., Herbin, M. \& Nevo, E. (1993). Ocular regression conceals adaptive progression of the visual system in the blind subterranean mammal. Nature 361, 156-159.

- De Vries, M.J. \& De Vries, A.J. (1995). A change in the pattern in circadian running-wheel activity after lesions of the intergeniculate leaflet and the ventral lateral geniculate nucleus in the Syberian hamster. Biol. Rhythm Res. 26, 311-340.

- Goel, N. \& Lee, T. (1995). Social cues accelerate reentrainment of circadian rhythms in diurnal female Octodon degus (Rodentia-Octodontidae).

Chronobiol. Int. 12, 311-323.

- Goldman, B.D. (1999). The circadian timing system and reproduction in mammals. Steroids 64, 679-685.

- Goldman, B.D., Goldman, S.L., Riccio, A.P. \& Terkel, J. (1997). Circadian patterns of locomotor activity and body temperature in blind mole-rats, Spalax ehrenbergi. J. Biol. Rhythms 12, 348-361.

- Haim, A., Heth, G., Pratt, H. \& Nevo, E. (1983). Photoperiodic effects on thermoregulation in a 'blind' subterranean mammal. J. Exp. Biol. 107, 59-64.

- Hart, L., Bennett, N.C., Malpaux, B., Chimimba, C.T. \& Oosthuizen, M.K. (2004). The chronobiology of the natal mole-rat, Cryptomys hottentotus natalensis. Physiol. Behav. 82, 563-569.

- Hickman, G.C. (1979). A live trap and trapping technique for fossorial mammals. S. Afr. J. Zool. 14, 9-12.

- Lovegrove, B.G., Heldmaier, G. \& Ruf, T. (1993). Circadian activity in colonies of 'blind' molerats, Cryptomys damarensis (Bathyergidae). S. Afr. Tydskr. Dierk. 28, 46-55.

- Lovegrove, B.G. \& Papenfus, M.E. (1995). Circadian activity rhythms in the solitary Cape molerat (Georychus capensis: Bathyergidae) with some evidence of splitting. Physiol. Behav. 58, 679-685.

- Moore, R.Y. (1983). Organization and function of a central nervous system circadian oscillator: the suprachiasmatic hypothalamic nucleus. Fed. Proc. 42, 2783-2789.

- Negroni, J., Bennett, N.C. \& Cooper, H.M. (2003). Organization of the circadian system in the subterranean mole rat, Cryptomys hottentotus (Bathyergidae). Brain Res. 967, 48-62. 
- Němec, P., Burda, H. \& Peichl, L. (2004). Subcortical visual system of the African mole-rat Cryptomys anselli: to see or not to see? Eur. J. Neurosci. 20, 757-768.

- Oosthuizen, M.K., Cooper, H.M. \& Bennett, N.C. (2003). Circadian rhythms of locomotor activity in solitary and social species of African mole-rats (family: Bathyergidae). J. Biol. Rhythms 18, 481-490.

- Oster, H., Avivi, A., Joel, A., Albrecht, U. \& Nevo, E. (2002). A switch from diurnal to nocturnal activity in S. ehrenbergi is accompanied by an uncoupling of light input and the circadian clock. Curr. Biol. 12, 1919-1922.

- Reuss, S. (1996). Components and connections of the circadian timing system in mammals. Cell Tissue Res. 285, 353-378.

- Roenneberg, T. \& Foster, R.G. (1997). Twilight times: light and the circadian system. Photochem. Photobiol. 66, 549-561.

- Schöttner, K., Oosthuizen, M.K., Broekman, M. \& Bennett, N.C. (2006). Circadian rhythms of locomotor activity in the Lesotho mole-rat, Cryptomys hottentotus subspecies from Sani pass, South Africa. Physiol. Behav. 89, 205212.

- Sharma, V.K. (2003). Adaptive significance of circadian clocks. Chronobiol. Int. 20, 901-919.

- Tobler, I., Hermann, M., Cooper, H.M., Negroni, J., Nevo, E. \& Achermann, P. (1998). Rest-activity rhythm of the blind mole rat Spalax ehrenbergi under different lighting conditions. Behav. Brain Res. 96, 173-183.

- Van Esseveldt, L.K.E., Lehman, M.N. \& Boer, G.J. (2000). The suprachiasmatic nucleus and the circadian time-keeping system revisited. Brain Res. Rev. 33, 34-77.

- Vasicek, C.A., Oosthuizen, M.K., Cooper, H.M. \& Bennett, N.C. (2005). Circadian rhythms of locomotor activity in the subterranean Mashona mole rat, Cryptomys darlingi. Physiol. Behav. 84, 181-191.

- Vuillez, P., Herbin, M., Cooper, H.M, Nevo, E. \& Pevet, P. (1994). Photic induction of Fos immunoreactivity in the suprachiasmatic nuclei of the blind mole rat (Spalax ehrenbergi). Brain Res. 654, 81-4. 